\title{
Further delineation of the phenotype of PAK3-associated x-linked intellectual disability: Identification of a novel missense mutation and review of literature
}

\section{Introduction}

$\mathrm{X}$-linked intellectual disability (XLID) accounts for approximately $5-16 \%$ of males with intellectual disability. It is estimated that at least 200 genes are implicated in XLID, and the approximately 170 XLID entities are clinically classified as syndromic or non-syndromic (Stevenson et al., 2009; Lubs et al., 2012).

The p21-activated kinase 3 (PAK3) gene was the fourth to be associated with non-syndromic XLID, type 30 (OMIM: \#300558) (Allen et al., 1998). PAK3 is a serine/threonine kinase and its sequence is highly conserved between species. The kinase acts as a downstream effector of Rac1 and Cdc42 Rho-GTPases and has important roles in actin cytoskeletal reorganization, dendritic spine morphology, density, stability and dynamics and also in synaptic currents (Kreis et al. 2007; Dubos et al. 2012; Thévenot et al., 2011).

Since 1998, nine different PAK3 mutations have been identified in 46 affected individuals from nine families of different ethnicity. Here, we report the first case of a Hungarian patient with intellectual disability associated with a novel PAK3 mutation and review the cases previously described in the literature.

\section{Clinical report}

The proband presented at genetic counselling at the age of 14 years with intellectual disability, autistic characteristics and behavioral problems.

He was born at term by spontaneous delivery following a normal pregnancy, with normal birth weight and length as a first child of Caucasian non-consanguineous parents. Autistic characteristics and delayed psychomotor development were first noted at the age of 3 years (Brunet-Lézine test: gross motor skills: 65; fine motor skills: 61; language skills: 58; sociability: 52; overall developmental quotient of 59). Special training was initiated. He started to speak and maintain eye contact at 4 years and let his mouth hang open with constant drooling until the age of 4.5 years. He was toilet-trained by the age of 5.5 years, but accidental soiling still happens.

He had three generalized tonic seizure episodes with fever in early childhood and experienced short absence-like episodes and unusual grimacing in the 1.5 year previous to examination. Baseline and sleepdeprived EEGs were repeatedly normal and brain MRI detected no abnormality. Temper tantrums and occasional aggressive behavior has been reported, but no sleep disturbance. At present, he receives risperidone treatment and attends special school.

On examination, he was cooperative, his body weight (43 kg, 10-25 percentile) and height $(158 \mathrm{~cm}, 25$ percentile) were normal. Microcephaly (Supplementary Table 1), mild thoracic kyphosis, dorsolumbar scoliosis, ankle valgus, pectus carinatum, wide-spaced nipples and spina bifida occulta with a sacral dimple were noted. His facial features included large ears, prominent but not bulbous nose, low forehead, downslanting palpebral fissures, thin upper lip and high-arched palate (Fig. 1A). His sexual maturation and testicular size were normal. Neurological examination revealed small muscle bulk in the limb-girdle muscles with normal tone and strength, mild postural and intentional tremor, symmetric brisk reflexes without spasticity and no gait disturbance.

Neuropsychological assessment showed mild-to-moderate intellectual disability with moderate impairment of visuo-spatial, reading, writing, comprehension and counting skills and severe attention deficit, mood imbalance, anxiety and autistic traits (WoodcockJohnson and Snijders-Oomen nonverbal intelligence tests: age equivalent of 5;2 and 5;3, respectively).

Quantitative and qualitative blood count, serum electrolytes, lactate, carbamide, uric acid, creatinine, creatine kinase and liver enzyme levels, inflammatory and autoinflammatory parameters, serum amino acid and acyl-carnitine profile, serum and urine dopamine and serotonin levels showed no marked discrepancy. Audiology detected mild sensorineural hearing loss, however, the examination was inconclusive due to lack of cooperation. On nephrological examination, underactive bladder function was detected. Abdominal ultrasound, echocardiography, ECG and ophthalmology showed no abnormality. Karyotyping on G-banded chromosomes using standard procedures detected no major aberration and testing for Fragile-X syndrome showed no triplet repeat expansion in FMR1.

No relatives had intellectual disability or dysmorphic facial features (Fig. 1B).

\section{Methods}

Genomic DNA was isolated from peripheral blood samples from the proband and his relatives using the Promega Maxwell ${ }^{\circledR}$ RSC Blood DNA Kit. Clinical exome analysis was carried out on the whole exome sequence obtained using Illumina NextSeq500 sequencer after library preparation with Roche KAPA HyperPrep library kit and SeqCap EZ MedExome capture kit.

Mean average depth of on-target coverage in the sequenced exome was 69X (target bases at 10x coverage: 96\%; at 20x coverage: 93\%; at 30x coverage: $86 \%$ ). Reads were aligned to the human reference genome (GRCh37) using BWA (v.0.7.12). Among 120,469 variants, deleterious ones were prioritized on the basis of the functional relevance of genes, inheritance models and minor allele frequency (MAF) in the general population (gnomAD and in-house databases). As a result of the filtering, a novel variant in the PAK3 gene was identified as the most probable pathogenic variant. The variant was submitted to a combination of 14 variant prediction tools and was confirmed by bidirectional Sanger sequencing (Supplementary Table 1).

PyMOL Molecular Graphics System (version 2.0 Schrödinger, LLC) 
A

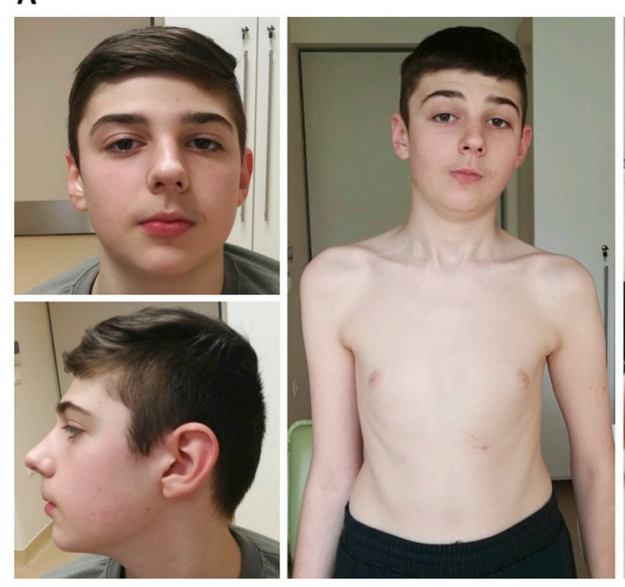

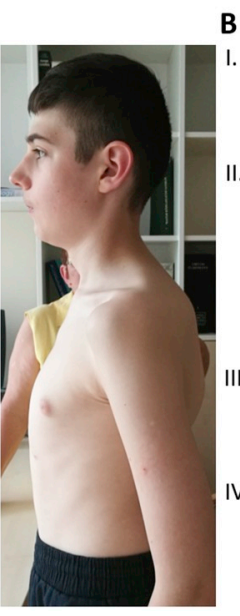

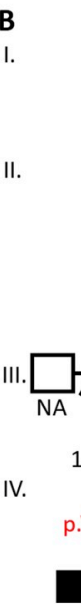

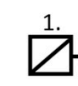
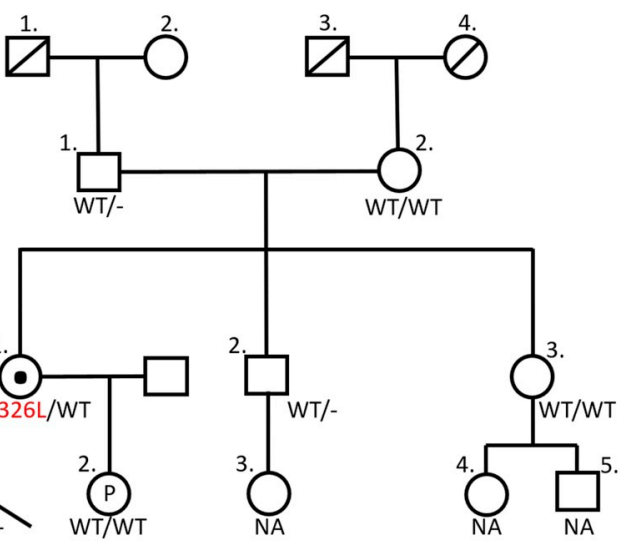

Affected $\bigodot x-l i n k e d$ carrier

Fig. 1. Pictures and pedigree of the patient.

(A) The images of the proband were captured at the age of 14 years (first column) and 14.5 years (second and third column). (B) The mother of the proband was pregnant with a female, non-carrier fetus, as confirmed by karyotyping and targeted mutation analysis. Proband is indicated with an arrow. NA: not assessed.
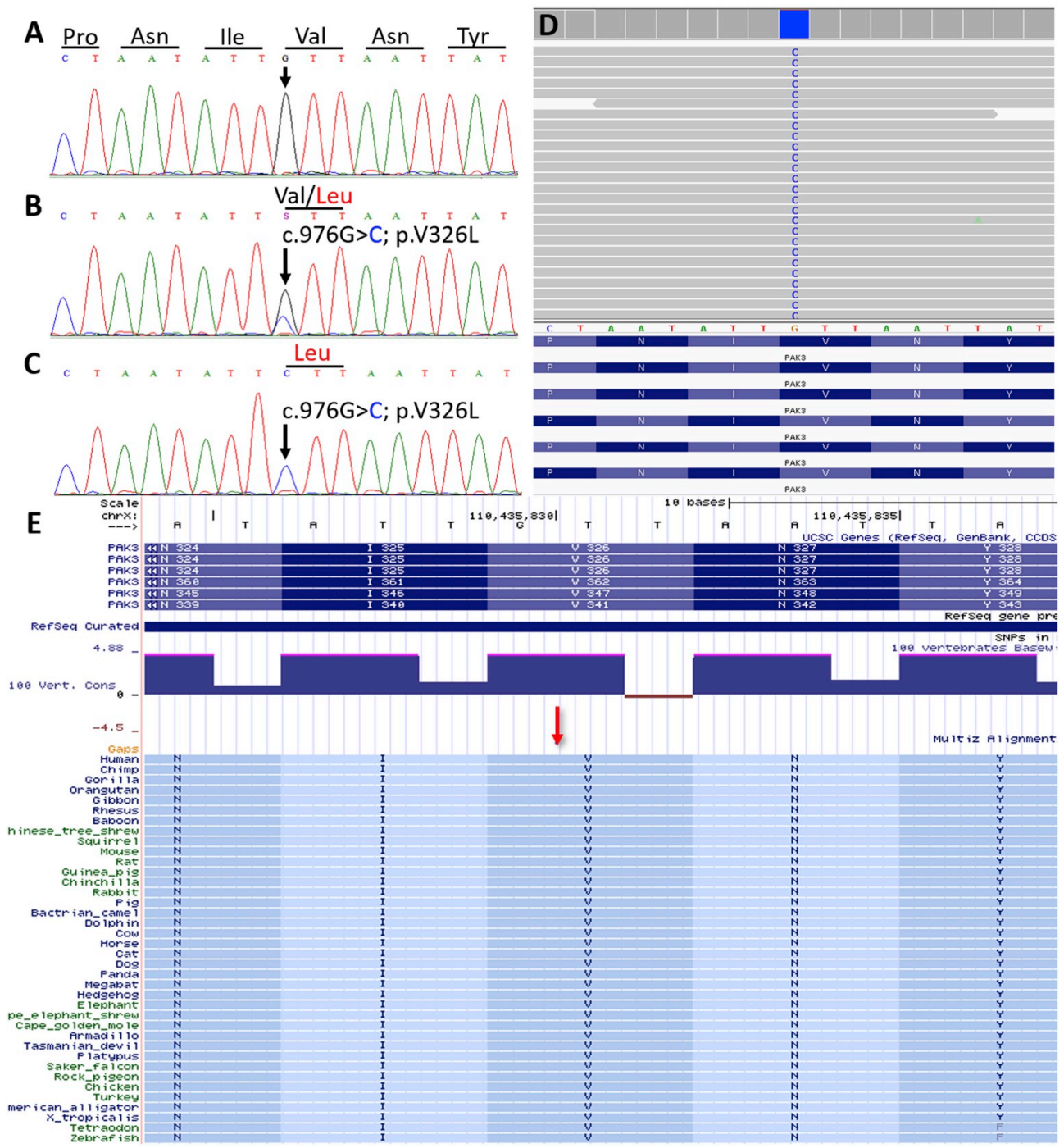

Fig. 2. Analysis of the novel Val326Leu PAK3 variant by clinical exome sequencing and bidirectional Sanger sequencing.

Electropherogram of the (A) wild-type sequence, (B) heterozygous female carrier (mother) and (C) hemizygous proband. (D) The screen shot from the Integrative Genomic Viewer shows part of the (51/51) reads supporting the c.976G > C variant in the proband. (E) Screen shot from the UCSC Genome Browser represents the high conservation of the amino acid residue in position 326 and surrounding genomic context. Arrows indicate the nucleotide change. 


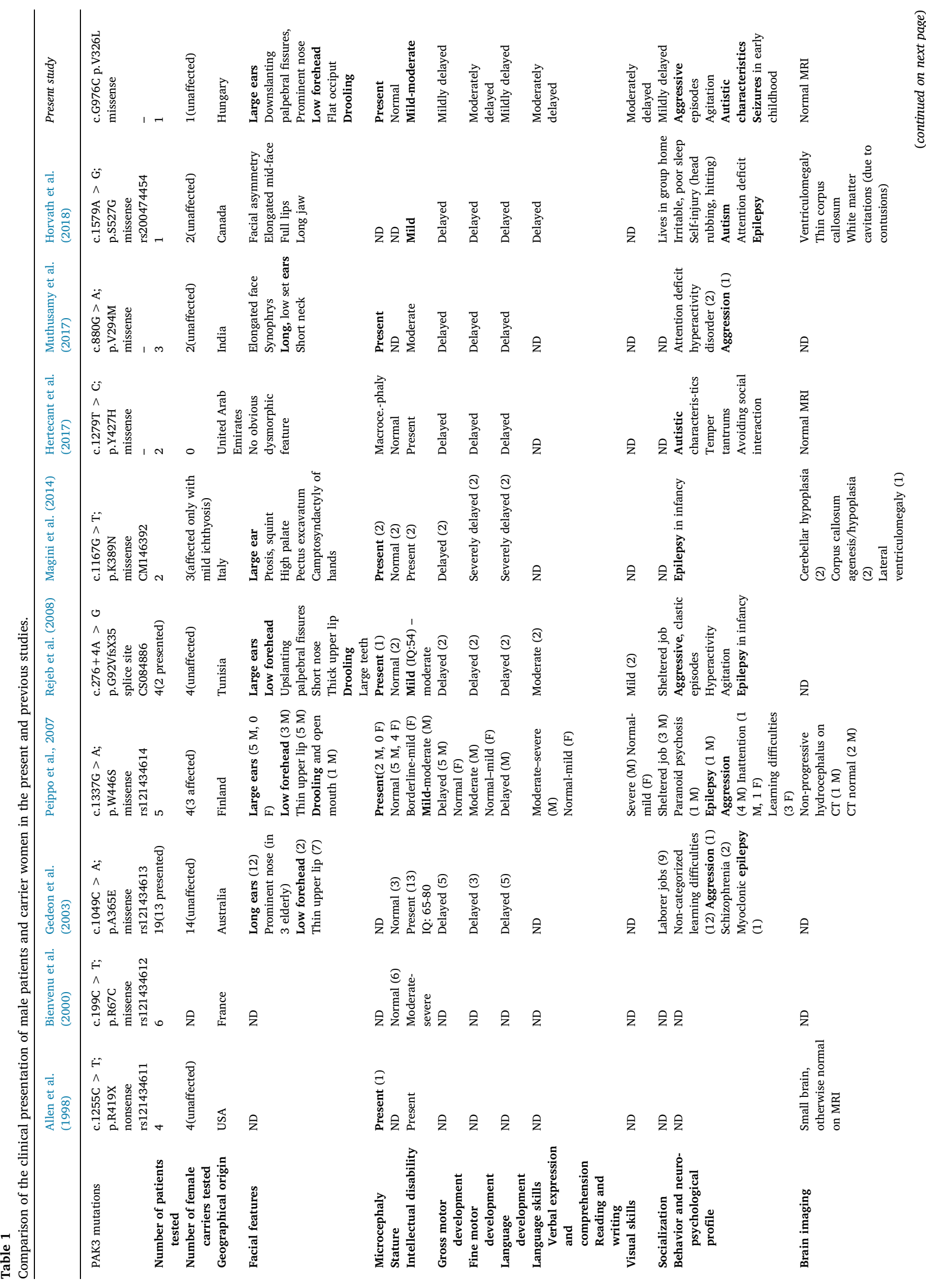


was used to evaluate in silico the changes in the mutant PAK3 protein structure. The wild-type three-dimensional protein structure has been obtained from RCSB Protein Data Bank (ID: 6fd3) and submitted to PyMOL's Wizard/Mutagenesis on protein application to create and visualize the specific mutant PAK3 protein.

Additional testing included maternity testing on the sample from the proband, maternity-paternity testing on the samples from the proband's mother and maternal grandparents (Promega PowerPlex ${ }^{\circledast}$ ESX 17 System) and X-chromosome inactivation assay (Supplementary Table 1) (Kiedrowski et al., 2011).

The results were assessed and classified according to the ACMG guideline (Supplementary Table 1) (Richards et al., 2015).

\section{Results}

One novel variant - NM_001128167.2:c.976G > C;p.(Val326Leu) (ClinVar submission number: SCV000927119; LOVD accession number: \#0000578234, DB-ID: PAK3_000063) - has been detected in exon 10 of $P A K 3$ gene, which is associated with $\mathrm{X}$-linked non-syndromic intellectual disability. The variant was present in the proband in a hemizygous form and in unaffected mother in a heterozygous form but not in any other healthy family members tested (Figs. 1B, Fig. 2A-D) or in the control databases (141,456 whole exome/genome sequences contained in gnomAD, in +500 exome sequences of the in-house database of qGenomics or in 151 exome sequences of Hungarian patients recruited in other projects).

The Val326Leu variant was predicted to be probably damaging by PANTHER and PolyPhen 2 and damaging by the other 12 prediction tools. The Val326Leu variant is located in the highly conserved protein kinase domain of the PAK3 gene (Fig. 2E).

The in silico modelling suggested that the wild-type residue $\mathrm{Val}^{326}$ is located on the surface of the ATP-binding recess of the kinase domain of PAK3 in close vicinity to the ATP molecule (at a distance of $4.0 \AA$ ); however, it does not bind to ATP. The amino acid change to Leu ${ }^{326}$ resulted in a shortening of the distance between the ATP molecule and residue 326 (3.6 $\AA$ ), a change in the surface area of the ATP-binding recess and the formation of a new hydrogen bond between residues Leu $^{326}$ and Leu ${ }^{403}$ (Supplementary Fig. 1), thus supporting its impact on protein structure and function.

Maternity and paternity testing revealed no discrepancy and, therefore, confirmed the de novo origin of the variant in the proband's mother.

Based on the ACMG criteria (Supplementary Table 1) and a detailed clinical comparison with previously described patients (Table 1), the results supported the ethiopathogenicity of the novel Val326Leu PAK3variant.

\section{Discussion}

To the best of our knowledge, this family is the first Hungarian and the tenth family reported worldwide with $P A K 3$-associated non-syndromic XLID. Until now, one nonsense, one splice site and seven missense mutations have been reported for the PAK3 gene. Eight of ten mutations are located in the kinase domain of the protein, presumably disabling its enzymatic function. The location of the $\mathrm{Leu}^{326}$ mutation in the kinase domain and the additional hydrogen bond formation suggests that it may influence the ATP-binding capacity and also the structure of the protein.

PAK3 function and regulation is complex. When activated by GTPbound Rho GTPases (Cdc42 and Rac1), PAK3 kinase phosphorylates other signaling molecules in neurons. The PAK3 function is important for the fine-wiring of the synaptic network in the brain. Therefore, lossof function mutations in the PAK3 gene are believed to lead to decreased neural plasticity and cognitive impairment without major structural brain abnormalities, also referred to as synaptopathies (Horvath et al., 2018). However, brain developmental abnormalities 
have been reported in some patients carrying variants of the PAK3 gene, which may be a result of PAK3 protein involvement in other signaling pathways (Magini et al., 2014).

In the current paper, we provide a thorough, comprehensive clinical review of $P A K 3$-patients described in the literature to date (Table 1 ), which allowed us to deduce the typical phenotypic features in PAK3XLID: microcephaly, mild-to-moderate intellectual disability in males, large ears, low frontal hairlines, elongated face, muscle hypotonia in infancy, drooling, seizures, aggression, anxiety and autistic behavior. In addition, this is the first reported patient who also has occult spina bifida and mild thoracolumbar deformity, however these findings are common in the general population and thus, may also be unrelated features.

Copy number variations in the PAK3-containing chromosomal region (Xq23) have also been reported in syndromic female patients with moderate-to-severe intellectual disability (Hoischen et al., 2009; Jin et al., 2015). However, these phenotypes are distinct from PAK3-XLID due to the haploinsufficiency of other genes involved.

Beside the genetic importance of the diagnosis of PAK3-associated $\mathrm{XLID}$, it may also have therapeutic consequence, as presented in a previous report (Horvath et al., 2018). Their patient had epilepsy, cerebral laceration as a result of early-onset, intractable, self-injurious behavior due to decreased levels of dopamine and serotonin metabolites in the cerebrospinal fluid. Low-dose replacement therapy drastically improved and stabilized his condition. It was hypothesized that PAK3 dysfunction may lead to diminished dendritic spines and consequentially diminished postsynaptic dopamine receptors or may impair the phosphorylation of the tyrosine hydroxylase, ultimately leading to decreased catecholamine synthesis (Horvath et al., 2018; Daubner et al., 2011). Thus, in case of behavioral or psychiatric deterioration, determination of the neurotransmitter levels and if necessary, supplementation may be considered. However further studies are needed for final recommendations.

In conclusion, our paper provides further insight into the genetic and phenotypic background of PAK3-XLID, expands the PAK3 mutation spectrum, and may help others with the genetic diagnosis by highlighting the common typical PAK3-associated features.

Funding

This work was funded from the GINOP-2.3.2-15-2016-00039 grant.

\section{Consent for participation and publication}

Written informed consent was obtained from the proband and family members for clinical and genetic testing using a consent form approved by the Ethics Review Committee, Faculty of Medicine, University of Szeged. The study was conducted according to the Principles of the Helsinki Declaration. Written informed consent for publication of the patient's clinical details and images was obtained from the proband's parent.

\section{Declaration of competing interest}

The authors have no conflict of interest to report.

\section{Acknowledgements}

We thank the family of the proband for the kind cooperation with this study, Zsuzsanna Horváth-Gárgyán, Blanka Godza, Dóra Isaszegi, Anikó Gárgyán for their skilled technical assistance, and Dr. Shannon
Frances for providing language help.

\section{Appendix A. Supplementary data}

Supplementary data to this article can be found online at https:// doi.org/10.1016/j.ejmg.2019.103800.

\section{References}

Allen, K.M., Gleeson, J.G., Bagrodia, S., et al., 1998. PAK3 mutation in nonsyndromic Xlinked mental retardation. Nat. Genet. 20 (1), 25-30.

Bienvenu, T., des Portes, V., McDonell, N., et al., 2000. Missense mutation in PAK3, R67C, causes X-linked nonspecific mental retardation. Am. J. Med. Genet. 93 (4), 294-298.

Daubner, S.C., Le, T., Wang, S., 2011. Tyrosine hydroxylase and regulation of dopamine synthesis. Arch. Biochem. Biophys. 508 (1), 1-12.

Dubos, A., Combeau, G., Bernardinelli, Y., et al., 2012. Alteration of synaptic network dynamics by intellectual disability protein PAK3. J. Neurosci. 32 (2), 519-527.

Gedeon, A.K., Nelson, J., Gécz, J., et al., 2003. X-linked mild non-syndromic mental retardation with neuropsychiatric problems and the missense mutation A365E in PAK3. Am. J. Med. Genet. 120A (4), 509-517.

Hertecant, J., Komara, M., Nagi, A., et al., 2017. A de novo mutation in the X-linked PAK3 gene is the underlying cause of intellectual disability and macrocephaly in monozygotic twins. Eur. J. Med. Genet. 60 (4), 212-216.

Horvath, G.A., Tarailo-Graovac, M., Bartel, T., et al., 2018. Improvement of self-injury with dopamine and serotonin replacement therapy in a patient with a hemizygous PAK3 mutation: a new therapeutic strategy for neuropsychiatric features of an intellectual disability syndrome. J. Child Neurol. 33 (1), 106-113.

Hoischen, A., Landwehr, C., Kabisch, S., et al., 2009. Array-CGH in unclear syndromic nephropathies identifies a microdeletion in Xq22.3-q23. Pediatr. Nephrol. 24, 1673-1681.

Jin, Z., Yuc, L., Geng, J., et al., 2015. A novel 47.2 Mb duplication on chromosomal bands Xq21.1-25 associated with mental retardation. Gene 567 (1), 98-102.

Kiedrowski, L.A., Raca, G., Laffin, J.J., et al., 2011. DNA methylation assay for X-chromosome inactivation in female human iPS cells. Stem Cell Rev. Rep. 7, 969-975.

Kreis, P., Thévenot, E., Rousseau, V., et al., 2007. The p21-activated kinase 3 implicated in mental retardation regulates spine morphogenesis through Cdc42-dependent pathway. J. Biol. Chem. 282 (29), 21497-21506.

Lubs, H.A., Stevenson, R.E., Schwartz, C.E., 2012. Fragile X and X-linked intellectual disability: four decades of discovery. Am. J. Hum. Genet. 90, 579-590.

Magini, P., Pippucci, T., Tsai, I.C., et al., 2014. A mutation in PAK3 with a dual molecular effect deregulates the RAS/MAPK pathway and drives an X-linked syndromic phenotype. Hum. Mol. Genet. 23 (13), 3607-3617.

Muthusamy, B., Selvan, L.D.N., Nguyen, T.T., et al., 2017. Next-generation sequencing reveals novel mutations in X-linked intellectual disability. OMICS 21 (5), 295-303.

Peippo, M.1, Koivisto, A.M., Särkämö, T., et al., 2007. PAK3 related mental disability: further characterization of the phenotype. Am. J. Med. Genet. 143A (20), 2406-2416.

Rejeb, I., Saillour, Y., Castelnau, L., et al., 2008. A novel splice mutation in PAK3 gene underlying mental retardation with neuropsychiatric features. Eur. J. Hum. Genet. 16 (11), 1358-1363.

Richards, S., Aziz, N., Bale, S., et al., 2015. Standards and guidelines for the interpretation of sequence variants: a joint consensus recommendation of the American college of medical genetics and genomics and the association for molecular pathology. Genet. Med. 17 (5), 405-424.

Stevenson, R.E., Schwartz, C.E., 2009. X-linked intellectual disability: unique vulnerability of the male genome. Dev. Disabil. Res. Rev. 15 (4), 361-368.

Thévenot, E., Moreau, A.W., Rousseau, V., et al., 2011. p21-Activated kinase 3 (PAK3) protein regulates synaptic transmission through its interaction with the Nck2/Grb4 protein adaptor. J. Biol. Chem. 286 (46), 40044-40059.

Dóra Nagy $^{\mathrm{a}, *}$, Katalin Farkas ${ }^{\mathrm{a}}$, Lluís Armengol ${ }^{\mathrm{b}}$, Maria Segura ${ }^{\mathrm{b}}$, Gloria Kafui Esi Zodanu ${ }^{\mathrm{a}}$, Bernadett Csányi ${ }^{\mathrm{c}}$, Alíz Zimmermann ${ }^{\mathrm{d}}$, Barbara Vámos ${ }^{\mathrm{d}}$, Márta Széll ${ }^{\mathrm{a}}$

${ }^{a}$ Department of Medical Genetics, Faculty of Medicine, University of Szeged, Szeged, Hungary

${ }^{\mathrm{b}}$ Quantitative Genomic Medicine Laboratories Ltd (qGenomics), Esplugues del Llobregat, Barcelona, Catalonia, Spain

${ }^{\mathrm{c}}$ Department of Forensic Medicine, Faculty of Medicine, University of Szeged, Szeged, Hungary

${ }^{\mathrm{d}}$ Department of Pediatrics and Pediatric Health Center, Faculty of Medicine, University of Szeged, Szeged, Hungary E-mail address: nagy.dora@med.u-szeged.hu (D. Nagy). 